\title{
Rapid Screening Tolerance of 19 Soybean Varieties to Drought in the Germination Phase
}

\author{
H. Pujiwati ${ }^{1 *}$, A. Romeida ${ }^{1}$, Widodo $^{1}$, W. Prameswari ${ }^{1}$, M. 1. Husna ${ }^{1,}$ \\ Anandyawati ${ }^{2}$,
}

\author{
${ }^{I}$ Departement of Crop Production, faculty of Agriculture, The University of Bengkulu, \\ W.R. Supratman, st. Kandang Limun, Bengkulu, 38371. \\ ${ }^{2}$ Departement of Soil Science, faculty of Agriculture, The University of Bengkulu, \\ W.R. Supratman, st. Kandang Limun, Bengkulu, 38371. \\ "Corresponding author email: hesti_pujiwati@unib.ac.id
}

\begin{abstract}
Drought stress is an inhibiting factor for plant growth and development. Drought stress reduces the seed to germinate and to grow on different varieties. Response to germination to drought stress is the beginning signal of drought tolerance for several varieties of plants. The purposes of this study were to evaluate the germination response of 16 soybean varieties, to determine the degree of drought stress tolerance of 19 soybean varieties, and to identify varieties that are tolerant as well as sensitive to drought which stimulated by polyethylene glycol (PEG). Soybean seeds were added to sand media containing PEG (6000) at concentrations of $0 \%, 5 \%, 10 \%, 15 \%$, and $20 \%$ which were equivalent to $0,-0.03 \mathrm{Mpa},-0.19 \mathrm{Mpa},-041$ and $067 \mathrm{Mpa}$ for 8 days. The germination responses by calculating the vigor index, germination, root length, hypocotyl length, root length and hypocotyl length ratio, fresh weight sprouts. Inhibition of germination increases with a reduction in water potential. Each soybean seed tested showed different germination responses which were different under drought stress conditions. The results of 19 varieties showed only one variety which was identified to be drought-tolerant, however, 9 varieties were identified as medium droughttolerant, and 9 varieties were identified as sensitive to drought. The variety that was tolerant to drought was Gepak Kuning. Varieties that were sensitive to drought were Dena 2, Dega 1, Wilis, Edamame, Devatra 1, Devatra 2, Grobogan, Anjasmoro, and Detam 1.
\end{abstract}

Keywords: drought stress, soybean, polyethylene glycol (PEG), tolerance

\section{INTRODUCTION}

Soybean (Glycine max. L.) is a food commodity that contains a source of protein and amino acid content such as mentioned, lipstick, and trypsin, so it is widely consumed to meet nutritional needs and foodstuffs for humans. Soybean seeds contain substances that are useful and needed by humans. The contents of soybeans are protein $(35 \%)$, carbohydrates $(35 \%)$, fat $(15 \%)$, water $(13 \%)$ [1].

Based on the Central Statistics Agency, it is stated that the national soybean production from year to year has fluctuated. Soybean production in 2016-2018 was 859,653 tons, 538,728 tons, and 982,598 tons. Meanwhile, soybean production in Bengkulu Province from 2016-2018 was 4,664 tons, 413 tons, and 3,477 tons [2]. The annual demand for soybeans reaches an average of 2.3 million tons, while domestic soybean production is only able to meet the needs of around 920 thousand - 1.035 million tons. able to meet the consumption needs of an average of $8.12 \mathrm{~kg} / \mathrm{capital}$ each year [3], so to meet the insufficient consumption of soybeans, we must import from America [4].

One of the obstacles to increasing soybean productivity in Indonesia is drought stress. Soybeans are generally planted on dry land or after the main food crop at the end of the rainy season so that water availability is often an obstacle.

Efforts made to overcome the problem of drought stress in soybean cultivation are by providing soybean varieties that are tolerant of drought stress through a plant breeding approach. Although drought stress is one of the limiting factors for soybean production, not many droughttolerant varieties have been released. Around 1000 soybean germplasm selection numbers in Indonesia, there are about 62 varieties that have been cultivated by farmers [5]. However, from this number, there is no information regarding the level of tolerance to drought stress. Strategis 
for screening varieties that are tolerant of drought stress are very difficult to implement in the field because it is difficult to provide homogeneous stresses and requires a long time. The ex vitro technique using polyethylene glycol (PEG) is a very effective method to study the effect of water stress on germination and sprout growth $[6],[7],[8]$ and is a simple and inexpensive method. to screen large amounts of germplasm in a short and accurate period [9], [6], [10], [11]. According to Muller and Whitsitt [12] and Mullahey et al. [13], polyethylene glycol is an osmotic compound that can be used to simulate drought stress conditions because PEG can reduce water potential and is not toxic to plants. Laboratory tests for screening of drought tolerance levels of genotypes show a significant correlation with drought tolerance levels in the field [14]. The ability to germinate under water stress simulated by PEG varies between plant genotypes [15],[16],[17],[18]. Pearl millet cultivars that were dry tolerant had a higher germination ability than sensitive cultivars because they absorbed less water for germination [19]. Results of screening on wheat by Guoth et al. [11] showed that the tolerant genotype had a smaller reduction in leaf water potential and a higher accumulation of ABA hormone compared to the sensitive genotype. This research was carried out by screening 19 varieties of soybean in the germination phase using PEG at different concentrations. This study aimed to evaluate the germination response of 19 soybean varieties stimulated by Polyethylene glycol (PEG), to determine the drought tolerance level of 16 soybean varieties based on the germination response in media containing PEG, and to identify drought-tolerant and sensitive varieties.

\section{MATERIALS AND METHODS}

The research was conducted at the Agronomy Laboratory of the Faculty of Agriculture, the University of Bengkulu in September 2020. The materials used were 19 varieties of soybeans, Polyethylene Glycol (PEG) 6000 , distilled water, frosted paper, plastic. The tools used were rulers, scissors, plastic trays, and stationery.

The research design used a completely randomized design which consists of 2 factors. The first factor is the soybean varieties which consist of: Detam 2, Dering 1, Deja 1, Detap 1, Detam 4, Anjasmoro, Devon 1, Dena 1, Dega 1, Grobongan, Derap 1, Detam 3, Detam 1, GepakKuning, Dena 2, Wilis, Edamame, Devatra 2, Devatra 1, The second factor is the PEG concentration consisting of $0 \%, 5 \%, 10 \%$, and $20 \%$. Each treatment combination was repeated 3 times to obtain 60 experimental units. Each experimental unit uses 25 seeds.

The seeds used are seeds that are not physically damaged, have the same size, shape, and color.

PEG 6000 was weighed according to the concentration treatment of each PEG (6000) at concentrations of $0 \%, 5 \%, 10 \%, 15 \%$, and $20 \%$ which were equivalent to $0,0.03 \mathrm{Mpa},-0.19 \mathrm{Mpa},-041$, and -
067 Mpa For 5 days, the weighted PEG concentration was put into a measuring cup, added with distilled water to 1 liter of PEG solution, then the solution was homogenized. Then the solution is poured into the tray according to the respective concentration treatment. Then the opaque paper is put into the tray containing the solution until all the paper is immersed, then drained until all the water gravity drops.

This study used the Rolled Paper Established on Plastic Test (UKDdp) method. Prepare 1 sheet of thin clear plastic measuring $20 \times 30 \mathrm{~cm}$, then spread it on the Table, then prepare 7 sheets of frosted paper, 4 sheets below, and 3 sheets of frosted paper on top of the seeds. 4 sheets of opaque paper media, then soak the paper in the tray containing the PEG solution according to the treatment, then drain and place on the prepared plastic. Furthermore, 25 seeds were arranged on paper for each experimental unit. The seeds are arranged in two rows, then covered again with 3 sheets of opaque paper, then rolled to the length of the substrate. The rolls are placed in jars and then stored in a germinator.

Germination is calculated using the formula:

$\sum$ sprout aged 5 days

$\sum$ planted seeds

The vigor index is calculated by the formula:

$\sum$ sprout aged 3 days

$\sum$ planted seeds

- Root length

- $\quad$ Length of hypocotyl

- The ratio of hypothetical length/root length

- $\quad$ Sprouts weight

The observed data were analyzed by using the analysis of variance using $\mathrm{F}$ count with a confidence level of $5 \%$, while the comparison between treatments was carried out using the Duncan Test (Duncan Multiple Range Test / DMRT).

The level of drought tolerance of soybean varieties was determined based on the drought sensitivity index (S) value of various germination variables. The tested soybean varieties are classified as drought-tolerant varieties if they have a value of $S<0.5$, including the medium group if 0.5 $\langle S\rangle 1$ and the drought-sensitive group if $S\rangle 1$ ). The sensitivity index is calculated using the Fischer and Maurer formula, 1978, as follows: drought stress. This is because each variety has a different response to the PEG 6000 concentration because each variety has a different ability to make osmotic adjustments to different PEG concentrations. This depends on the activity of genes in regulating the osmotic pressure and producing dissolved compounds to maintain balance in cells [20].

The results showed that the PEG concentration had a very significant effect on the vigor of the growing strength of soybean seeds, this was because the germination process required water, the function of this water absorption was to soften the seed coat and cause embryo and endosperm development, which caused the 
seed coat to break and experience imbibition to encourage the formation of hydrolysis enzymes such as $\alpha$-amylase, protease, ribonuclease, $\beta$-glyconase and phosphatase enzymes. These enzymes will diffuse into the endosperm catalyze food reserves into sugars, amino acids, during germination

\section{$(1-\mathrm{X} / \mathrm{Xp})$}

$\mathrm{Y}=$ average observed value for one particular variety under stress conditions

$\mathrm{Yp}=$ average observed value for one particular variety under non-stress conditions

$\mathrm{X}=$ mean value of observations for all varieties under stress conditions

$\mathrm{Xp}=$ average observed value for all varieties in nonstress conditions

If $\mathrm{S}<0.5=$ tolerant, $0.5<\mathrm{S}>1.0=$ medium and $\mathrm{S}>1.0=$ sensitive

\section{RESULTS AND DISCUSSION}

The addition of PEG to the germination medium is used to simulate drought stress conditions because PEG is able to bind water so that it can reduce water potential $\left(\Psi_{\mathrm{w}}\right)$ in the growing medium. Decreasing $\Psi_{\mathrm{W}}$ in the germination medium can inhibit the germination and growth of soybean sprouts. The higher the $\Psi_{\mathrm{w}}$ reduction in the growing medium the higher the inhibition of soybean germination (Table 1). The ex vitro technique using polyethylene glycol (PEG) is a very effective method to study the effect of water stress on germination and sprout growth [6], [7], [8] and is a simple and inexpensive method. to screen large amounts of germplasm in a short and accurate time period [9],[6],[10],[11].

The results of the analysis of variance ( $F$ test) in Table 1 and Table 2 show that black soybean varieties have a very significant effect on the observed variables. The higher the PEG concentration given, the lower the vigor index, the fresh weight of sprouts, the percentage of germination, hypocotyl length, root length, root length/hypocotyl length ratio. The ability to germinate for each variety varies with the level of metabolic process of the seeds will be disturbed, thus affecting seed germination [21]. The vigor index of Gepak Kuning variety in water stress - 0.67 was significantly different from Mpa compared to other varieties by 0.97 .

Table 1. Effect of drought stress on vigor index, fresh weight of sprouts and germination percentage of 19 soybean varieties

\begin{tabular}{|c|c|c|c|c|c|c|c|c|c|c|c|c|c|c|c|c|}
\hline \multirow[b]{2}{*}{ No } & \multirow{2}{*}{$\begin{array}{l}\text { Soybean } \\
\text { variety }\end{array}$} & \multicolumn{5}{|c|}{ Vigor Index } & \multicolumn{5}{|c|}{ Wet Germination Weight (g) } & \multicolumn{5}{|c|}{ Germination Percentage (\%) } \\
\hline & & 0 MPa & - 0.03 MPa & - 0.19 MPa & $\begin{array}{c}-0.41 \\
\text { MPa }\end{array}$ & - 0,67 MPa & 0 MPa & $\begin{array}{c}-0.03 \\
\text { MPa }\end{array}$ & $\begin{array}{c}-0.19 \\
\text { MPa }\end{array}$ & $\begin{array}{c}-0.41 \\
\text { MPa }\end{array}$ & $\begin{array}{c}-0,67 \\
\text { MPa }\end{array}$ & 0 MPa & $\begin{array}{c}-0.03 \\
\mathrm{MPa}\end{array}$ & $\begin{array}{c}-0.19 \\
\text { MPa }\end{array}$ & $\begin{array}{c}-0.41 \\
\text { MPa }\end{array}$ & - 0,67 MPa \\
\hline 1 & Grobogan & $1.00 \mathrm{a}$ & $1.00 \mathrm{a}$ & $1.00 \mathrm{a}$ & $0.625 \mathrm{e}$ & $0.25 \mathrm{fgh}$ & $0.935 \mathrm{bcde}$ & $0.7 \mathrm{f}$ & $0.74 \mathrm{~b}$ & $0.54 \mathrm{e}$ & $0.43 \mathrm{a}$ & $100 \mathrm{a}$ & $100 \mathrm{a}$ & $97.5 \mathrm{a}$ & $100 \mathrm{a}$ & $90 \mathrm{abc}$ \\
\hline 2 & Derap 1 & $1.00 \mathrm{a}$ & $0.95 a b c$ & $0.975 \mathrm{a}$ & $0.7 \mathrm{de}$ & $0.55 \mathrm{cdef}$ & $0.97 \mathrm{bcd}$ & $0.72 \mathrm{~d}$ & $0.72 \mathrm{c}$ & 0.441 & $0.44 \mathrm{c}$ & $100 \mathrm{a}$ & $90 \mathrm{~b}$ & $97.5 \mathrm{a}$ & $97.5 \mathrm{a}$ & $97.5 \mathrm{ab}$ \\
\hline 3 & Devatra 1 & $1.00 \mathrm{a}$ & $1.00 \mathrm{a}$ & $1.00 \mathrm{a}$ & 0.75 cde & 0.8 abcd & $1.23 \mathrm{a}$ & $1.11 \mathrm{~b}$ & $0.61 \mathrm{~h}$ & $2.71 \mathrm{a}$ & $0.36 \mathrm{k}$ & $100 \mathrm{a}$ & $100 \mathrm{a}$ & $100 \mathrm{a}$ & $88 \mathrm{~b}$ & $100 \mathrm{a}$ \\
\hline 4 & Detam 1 & $1.00 \mathrm{a}$ & $0.825 \mathrm{~cd}$ & $0.825 \mathrm{bc}$ & $0.225 \mathrm{f}$ & $0.15 \mathrm{gh}$ & $0.72 \mathrm{i}$ & $0.49 \mathrm{o}$ & $0.38 \mathrm{p}$ & $0.31 \mathrm{p}$ & $0.31 \mathrm{n}$ & $100 \mathrm{a}$ & $85 \mathrm{c}$ & $100 \mathrm{a}$ & $87.5 \mathrm{~b}$ & $70 \mathrm{de}$ \\
\hline 5 & Detam 3 & $1.00 \mathrm{a}$ & $1.00 \mathrm{a}$ & $0.975 \mathrm{a}$ & $0.925 \mathrm{abc}$ & $0.85 \mathrm{abc}$ & $0.73 \mathrm{hi}$ & $0.46 \mathrm{p}$ & $0.54 \mathrm{k}$ & 0.38 o & 0.341 & $100 \mathrm{a}$ & $100 \mathrm{a}$ & $97.5 \mathrm{a}$ & $97.5 \mathrm{a}$ & $100 \mathrm{a}$ \\
\hline 6 & $\begin{array}{c}\text { Gepak } \\
\text { Kuning }\end{array}$ & $1.00 \mathrm{a}$ & $1.00 \mathrm{a}$ & $0.95 \mathrm{ab}$ & $0.975 \mathrm{ab}$ & $0.975 \mathrm{a}$ & 0.75 ghi & $0.55 \mathrm{~m}$ & $0.39 \mathrm{o}$ & $0.28 \mathrm{r}$ & $0.29 \mathrm{o}$ & $100 \mathrm{a}$ & $100 \mathrm{a}$ & $100 \mathrm{a}$ & $100 \mathrm{a}$ & $95 \mathrm{ab}$ \\
\hline 7 & Dena 2 & $1.00 \mathrm{a}$ & $0.775 \mathrm{~d}$ & $0.675 \mathrm{~d}$ & $0.25 \mathrm{f}$ & 0.45 defg & 0.9 cdef & $0.64 \mathrm{i}$ & $0.49 \mathrm{~m}$ & $0.4 \mathrm{n}$ & $0.30 \mathrm{j}$ & $100 \mathrm{a}$ & $85 \mathrm{c}$ & $77.5 \mathrm{~b}$ & $77.5 \mathrm{c}$ & $70 \mathrm{de}$ \\
\hline 8 & Willis & $1.00 \mathrm{a}$ & $0.83 \mathrm{bcd}$ & $0.8 \mathrm{~cd}$ & $0.00 \mathrm{~g}$ & $0.2 \mathrm{fgh}$ & $0.47 \mathrm{j}$ & $0.38 \mathrm{q}$ & $0.43 n$ & $0.29 \mathrm{q}$ & $0.26 \mathrm{p}$ & $100 \mathrm{a}$ & $100 \mathrm{a}$ & $100 \mathrm{a}$ & $50 \mathrm{~d}$ & $80 \mathrm{~cd}$ \\
\hline 9 & \begin{tabular}{|l|} 
Devatra 2 \\
\end{tabular} & $1.00 \mathrm{a}$ & $0.83 \mathrm{bcd}$ & $0.8 \mathrm{~cd}$ & $1.00 \mathrm{a}$ & 0.6 abcdef & $1.04 \mathrm{~b}$ & $0.68 \mathrm{~g}$ & $0.62 \mathrm{~g}$ & $0.47 \mathrm{j}$ & $0.38 \mathrm{i}$ & $100 \mathrm{a}$ & $83 \mathrm{~d}$ & $80 \mathrm{~b}$ & $100 \mathrm{a}$ & $60 \mathrm{e}$ \\
\hline 10 & Dering 1 & $1.00 \mathrm{a}$ & $0.975 \mathrm{ab}$ & $0.925 \mathrm{abc}$ & 0.8 bcde & $0.95 \mathrm{ab}$ & $0.71 \mathrm{i}$ & $0.59 \mathrm{k}$ & $0.6 \mathrm{i}$ & $0.42 \mathrm{~m}$ & $0.33 \mathrm{~m}$ & $100 \mathrm{a}$ & $100 \mathrm{a}$ & $100 \mathrm{a}$ & $100 \mathrm{a}$ & $97.5 \mathrm{ab}$ \\
\hline 11 & Detam 2 & $1.00 \mathrm{a}$ & $0.925 \mathrm{abc}$ & $0.9 a b c$ & $0.85 \mathrm{abcd}$ & 0.7 abcde & $0.735 \mathrm{hi}$ & $0.66 \mathrm{~h}$ & $0.62 \mathrm{~g}$ & $0.55 \mathrm{~d}$ & $0.43 \mathrm{~d}$ & $100 \mathrm{a}$ & $100 \mathrm{a}$ & $97.5 \mathrm{a}$ & $100 \mathrm{a}$ & $95 \mathrm{ab}$ \\
\hline 12 & Deja 1 & $1.00 \mathrm{a}$ & $0.95 \mathrm{abc}$ & $1.00 \mathrm{a}$ & $0.925 \mathrm{abc}$ & 0.775 abcd & 0.875 cdefg & $0.71 \mathrm{e}$ & $0.64 \mathrm{f}$ & $0.52 \mathrm{~g}$ & $0.41 \mathrm{f}$ & $100 \mathrm{a}$ & $100 \mathrm{a}$ & $100 \mathrm{a}$ & $100 \mathrm{a}$ & $100 \mathrm{a}$ \\
\hline 13 & Dega 1 & $1.00 \mathrm{a}$ & $1.00 \mathrm{a}$ & $1.00 \mathrm{a}$ & $0.75 \mathrm{cde}$ & 0.325 efgh & $0.995 \mathrm{bc}$ & $0.8 \mathrm{c}$ & $0.65 \mathrm{e}$ & $0.57 \mathrm{c}$ & $0.42 \mathrm{e}$ & $100 \mathrm{a}$ & $100 \mathrm{a}$ & $100 \mathrm{a}$ & $92.5 \mathrm{ab}$ & $95 \mathrm{ab}$ \\
\hline 14 & Detam 4 & $1.00 \mathrm{a}$ & 0.9 abcd & $0.8 \mathrm{~cd}$ & $0.6 \mathrm{e}$ & 0.375 efgh & 0.765 fghi & $0.61 \mathrm{j}$ & $0.58 \mathrm{j}$ & $0.42 \mathrm{~m}$ & $0.39 \mathrm{~h}$ & $100 \mathrm{a}$ & $90 \mathrm{~b}$ & $85 \mathrm{~b}$ & $92.5 \mathrm{ab}$ & $87.5 \mathrm{abc}$ \\
\hline 15 & Detap 1 & $1.00 \mathrm{a}$ & $0.975 \mathrm{ab}$ & $1.00 \mathrm{a}$ & $0.75 \mathrm{cde}$ & 0.35 efgh & $0.985 \mathrm{bc}$ & $0.64 \mathrm{i}$ & $0.64 \mathrm{f}$ & $0.53 \mathrm{f}$ & $0.42 \mathrm{e}$ & $100 \mathrm{a}$ & $100 \mathrm{a}$ & $100 \mathrm{a}$ & $97.5 \mathrm{a}$ & $92.5 \mathrm{abc}$ \\
\hline 16 & Anjasmoro & $1.00 \mathrm{a}$ & $0.875 \mathrm{abcd}$ & $0.925 \mathrm{abc}$ & $0.675 \mathrm{de}$ & 0.325 efgh & 0.865 cdefgh & $0.66 \mathrm{~h}$ & $0.66 \mathrm{~d}$ & $0.46 \mathrm{k}$ & $0.4 \mathrm{~g}$ & $100 \mathrm{a}$ & $80 \mathrm{e}$ & $95 \mathrm{a}$ & $95 \mathrm{ab}$ & $85 \mathrm{bc}$ \\
\hline 17 & Devon 1 & $1.00 \mathrm{a}$ & $0.975 \mathrm{ab}$ & $0.925 \mathrm{abc}$ & $0.75 \mathrm{cde}$ & 0.575 bcdef & 0.84 defghi & 0.581 & $0.66 \mathrm{~d}$ & $0.49 \mathrm{i}$ & $0.46 \mathrm{~b}$ & $100 \mathrm{a}$ & $100 \mathrm{a}$ & $95 \mathrm{a}$ & $95 \mathrm{ab}$ & $95 \mathrm{ab}$ \\
\hline 18 & Dena 1 & $1.00 \mathrm{a}$ & $0.975 \mathrm{ab}$ & $0.975 \mathrm{a}$ & 0.75 cde & $0.55 \mathrm{cdef}$ & 0.815 efghi & $0.53 \mathrm{n}$ & 0.521 & $0.5 \mathrm{~h}$ & $0.43 \mathrm{~d}$ & $100 \mathrm{a}$ & $100 \mathrm{a}$ & $100 \mathrm{a}$ & $95 \mathrm{ab}$ & $100 \mathrm{a}$ \\
\hline
\end{tabular}


Table 2. Effect of drought stress on hypocotyl length, root length and ratio of hypocotyl length / seedling root length in 19 soybean varieties

\begin{tabular}{|c|c|c|c|c|c|c|c|c|c|c|c|c|c|c|c|c|}
\hline \multirow{2}{*}{ No } & \multirow{2}{*}{$\begin{array}{l}\text { Soybean } \\
\text { variety }\end{array}$} & \multicolumn{5}{|c|}{ Length of hypocotyl (cm) } & \multicolumn{5}{|c|}{ Length of root (cm) } & \multicolumn{5}{|c|}{$\begin{array}{c}\text { The ratio of hypothetical length/root } \\
\text { length }\end{array}$} \\
\hline & & 0 MPa & $\begin{array}{l}-0.03 \\
\text { MPa }\end{array}$ & $\begin{array}{l}-0.19 \\
\text { MPa }\end{array}$ & $\begin{array}{l}-0.41 \\
\text { MPa }\end{array}$ & $\begin{array}{l}-0,67 \\
\text { MPa }\end{array}$ & $0 \mathrm{MPa}$ & $\begin{array}{l}-0.03 \\
\text { MPa }\end{array}$ & $\begin{array}{l}-0.19 \\
\text { MPa }\end{array}$ & $\begin{array}{l}-0.41 \\
\text { MPa }\end{array}$ & $\begin{array}{l}-0,67 \\
\text { MPa }\end{array}$ & O MPa & $\begin{array}{l}-0.03 \\
\text { MPa }\end{array}$ & $\begin{array}{l}-0.19 \\
\text { MPa }\end{array}$ & $\begin{array}{l}-0.41 \\
\text { MPa }\end{array}$ & $\begin{array}{l}-0,67 \\
\text { MPa }\end{array}$ \\
\hline 1 & Grobogan & $8.08 \mathrm{~d}$ & $4.41 \mathrm{~m}$ & $4.1 \mathrm{~m}$ & $1.79 \mathrm{~m}$ & $0.88 \mathrm{p}$ & $13.14 \mathrm{bcd}$ & $12.73 \mathrm{f}$ & $14.98 \mathrm{c}$ & $13.31 \mathrm{c}$ & $10.36 \mathrm{~h}$ & $0.61 \mathrm{de}$ & $0.35 \mathrm{p}$ & $0.27 \mathrm{p}$ & $0.13 \mathrm{~h}$ & $0.08 \mathrm{~m}$ \\
\hline 2 & Derap 1 & $9.60 \mathrm{~cd}$ & $6.24 \mathrm{~h}$ & $4.58 \mathrm{j}$ & $1.43 \mathrm{o}$ & $1.23 \mathrm{~m}$ & $12.94 \mathrm{cde}$ & $12.83 \mathrm{e}$ & $13.17 \mathrm{f}$ & 9.531 & $13.49 \mathrm{~b}$ & $0.74 \mathrm{bcd}$ & $0.49 \mathrm{k}$ & $0.35 \mathrm{~m}$ & $0.15 \mathrm{gh}$ & 0.091 \\
\hline 3 & Devatra 1 & $15.88 \mathrm{a}$ & $12.00 \mathrm{a}$ & 4.181 & $3.05 \mathrm{f}$ & 1.241 & $21.06 \mathrm{a}$ & $17.5 \mathrm{a}$ & $7.8 \mathrm{o}$ & $10.18 \mathrm{k}$ & $6.76 n$ & $0.75 \mathrm{bcd}$ & $0.69 \mathrm{~g}$ & 0.361 & $0.3 \mathrm{~d}$ & $0.18 \mathrm{f}$ \\
\hline 4 & Detam 1 & $8.12 \mathrm{~d}$ & $3.86 \mathrm{q}$ & $2.32 \mathrm{r}$ & $0.9 \mathrm{p}$ & $0.79 \mathrm{q}$ & $5.61 \mathrm{i}$ & $6.33 \mathrm{q}$ & $4.67 \mathrm{q}$ & $4.51 \mathrm{~s}$ & $4.91 \mathrm{p}$ & $1.45 \mathrm{a}$ & $0.61 \mathrm{~h}$ & $0.5 \mathrm{~g}$ & $0.23 \mathrm{e}$ & $0.16 \mathrm{~g}$ \\
\hline 5 & Detam 3 & $12.84 \mathrm{bc}$ & $4.97 \mathrm{i}$ & $6.05 \mathrm{~g}$ & $2.58 \mathrm{~g}$ & $1.91 \mathrm{~d}$ & $14.75 \mathrm{bc}$ & $11.96 \mathrm{~h}$ & $15.26 \mathrm{~b}$ & $12.42 \mathrm{~d}$ & $12.79 \mathrm{c}$ & $0.85 \mathrm{bcd}$ & $0.42 \mathrm{n}$ & $0.4 \mathrm{k}$ & $0.21 \mathrm{e}$ & $015 \mathrm{~h}$ \\
\hline 6 & \begin{tabular}{|l|} 
Gepak \\
Kuning
\end{tabular} & $15.2 \mathrm{ab}$ & $9.2 \mathrm{c}$ & $7.27 \mathrm{a}$ & $4.09 \mathrm{~b}$ & $3.48 \mathrm{a}$ & $10.17 \mathrm{gh}$ & $10.58 \mathrm{k}$ & $14.11 \mathrm{~d}$ & $11.18 \mathrm{~h}$ & $13.66 \mathrm{a}$ & $1.49 \mathrm{a}$ & $0.87 \mathrm{c}$ & $0.52 \mathrm{f}$ & $0.32 \mathrm{~cd}$ & $0.25 \mathrm{~b}$ \\
\hline 7 & Dena 2 & $8.94 \mathrm{~d}$ & $4.73 \mathrm{j}$ & $2.56 \mathrm{q}$ & $1.46 \mathrm{n}$ & $1.03 \mathrm{n}$ & $5.65 \mathrm{i}$ & $5.57 \mathrm{r}$ & $6.39 \mathrm{p}$ & $4.68 \mathrm{r}$ & $4.95 \mathrm{o}$ & $1.58 \mathrm{a}$ & $0.85 \mathrm{~d}$ & $0.4 \mathrm{k}$ & $0.31 \mathrm{~cd}$ & $0.21 \mathrm{~d}$ \\
\hline 8 & Willis & $3.92 \mathrm{e}$ & $2.95 \mathrm{~s}$ & $3.00 \mathrm{p}$ & $0.78 \mathrm{q}$ & $0.6 \mathrm{r}$ & $2.88 \mathrm{j}$ & $2.87 \mathrm{~s}$ & $4.66 \mathrm{r}$ & $5.00 \mathrm{q}$ & $4.55 \mathrm{r}$ & $1.36 \mathrm{a}$ & $1.03 \mathrm{a}$ & $0.64 \mathrm{~b}$ & $0.16 \mathrm{gh}$ & $0.13 \mathrm{j}$ \\
\hline 9 & Devatra 2 & $10.82 \mathrm{~cd}$ & $7.52 \mathrm{~d}$ & $6.64 \mathrm{c}$ & 1.941 & $1.33 \mathrm{j}$ & $14.88 \mathrm{~b}$ & $9.14 \mathrm{n}$ & $7.8 \mathrm{o}$ & $13.32 \mathrm{~b}$ & $7.33 \mathrm{~m}$ & $0.73 \mathrm{bcd}$ & $0.82 \mathrm{e}$ & $0.85 \mathrm{a}$ & $0.15 \mathrm{gh}$ & $0.18 \mathrm{f}$ \\
\hline 10 & Dering 1 & $9.7 \mathrm{~cd}$ & $6.92 \mathrm{f}$ & $7.00 \mathrm{~b}$ & $3.44 \mathrm{~d}$ & $1.81 \mathrm{e}$ & $12.34 \mathrm{def}$ & $12.64 \mathrm{~g}$ & 11.031 & $11.56 \mathrm{~g}$ & 9.001 & $0.79 \mathrm{bcd}$ & $0.55 \mathrm{j}$ & $0.63 \mathrm{c}$ & $0.3 \mathrm{~d}$ & $0.2 \mathrm{e}$ \\
\hline 11 & Detam 2 & $9.86 \mathrm{~cd}$ & $7.21 \mathrm{e}$ & $6.3 \mathrm{e}$ & $6.36 \mathrm{a}$ & $2.55 \mathrm{~b}$ & $13.78 \mathrm{bcd}$ & $13.18 \mathrm{~d}$ & $15.34 \mathrm{a}$ & $16.03 \mathrm{a}$ & $10.99 \mathrm{e}$ & $0.71 \mathrm{bcd}$ & $0.55 \mathrm{j}$ & $0.41 \mathrm{j}$ & $0.4 \mathrm{a}$ & $0.23 \mathrm{c}$ \\
\hline 12 & Deja 1 & $9.63 \mathrm{~cd}$ & $10.2 \mathrm{~b}$ & $6.28 \mathrm{f}$ & $3.65 \mathrm{c}$ & $1.52 \mathrm{~g}$ & $13.38 \mathrm{bcd}$ & $10.84 \mathrm{j}$ & $13.14 \mathrm{~g}$ & $12.27 \mathrm{e}$ & $10.3 \mathrm{i}$ & $0.72 \mathrm{bcd}$ & $0.94 \mathrm{~b}$ & $0.48 \mathrm{i}$ & $0.3 \mathrm{~d}$ & $0.15 \mathrm{~h}$ \\
\hline 13 & Dega 1 & $8.50 \mathrm{~d}$ & $4.64 \mathrm{k}$ & $3.33 \mathrm{o}$ & 1.941 & $0.97 \mathrm{o}$ & $12.55 \mathrm{def}$ & $11.19 \mathrm{i}$ & $12.82 \mathrm{~h}$ & $11.74 \mathrm{f}$ & $9.61 \mathrm{j}$ & $0.67 \mathrm{cde}$ & $0.41 \mathrm{o}$ & $0.26 \mathrm{q}$ & $0.17 \mathrm{fg}$ & $0.1 \mathrm{k}$ \\
\hline 14 & Detam 4 & $10.08 \mathrm{~cd}$ & $6.72 \mathrm{~g}$ & $6.44 \mathrm{~d}$ & $2.24 \mathrm{j}$ & $2.34 \mathrm{c}$ & $10.67 \mathrm{fgh}$ & $9.42 \mathrm{~m}$ & $11.15 \mathrm{k}$ & $10.6 \mathrm{i}$ & $11.46 \mathrm{~d}$ & $0.94 \mathrm{~b}$ & $0.71 \mathrm{f}$ & $0.58 \mathrm{~d}$ & $0.21 \mathrm{e}$ & $0.2 \mathrm{e}$ \\
\hline 15 & Detap 1 & $10.35 \mathrm{~cd}$ & $3.92 \mathrm{o}$ & $4.56 \mathrm{k}$ & $2.37 \mathrm{i}$ & $1.27 \mathrm{k}$ & $11.91 \mathrm{defg}$ & $16.1 \mathrm{~b}$ & $14.04 \mathrm{e}$ & $10.48 \mathrm{j}$ & $13.49 \mathrm{~b}$ & $0.86 \mathrm{bc}$ & $0.25 \mathrm{r}$ & $0.32 \mathrm{o}$ & $0.23 \mathrm{e}$ & $0.13 \mathrm{j}$ \\
\hline 16 & Anjasmoro & $8.92 \mathrm{~d}$ & 4.451 & $4.59 \mathrm{i}$ & $2.45 \mathrm{~h}$ & $1.56 \mathrm{f}$ & 11.10 efg & 10.371 & $11.36 \mathrm{j}$ & $7.17 \mathrm{o}$ & $4.62 \mathrm{q}$ & $0.8 \mathrm{bcd}$ & $0.43 \mathrm{~m}$ & $0.4 \mathrm{k}$ & $0.34 \mathrm{bc}$ & $0.34 \mathrm{a}$ \\
\hline 17 & Devon 1 & $8.81 \mathrm{~d}$ & $3.65 \mathrm{r}$ & $3.67 \mathrm{n}$ & $1.99 \mathrm{k}$ & $1.35 \mathrm{i}$ & $12.25 \mathrm{def}$ & $8.27 \mathrm{o}$ & $11.02 \mathrm{~m}$ & $8.75 \mathrm{~m}$ & $10.42 \mathrm{~g}$ & $0.71 \mathrm{bcd}$ & 0.441 & $0.33 \mathrm{n}$ & $0.23 \mathrm{e}$ & $0.13 \mathrm{j}$ \\
\hline 18 & Dena 1 & $10.35 \mathrm{~cd}$ & $3.92 \mathrm{p}$ & $4.74 \mathrm{~h}$ & $3.1 \mathrm{e}$ & $1.5 \mathrm{~h}$ & $10.79 \mathrm{fgh}$ & $6.28 \mathrm{p}$ & $9.72 n$ & $8.51 \mathrm{n}$ & $10.73 \mathrm{f}$ & $0.96 \mathrm{~b}$ & $0.57 \mathrm{i}$ & $0.49 \mathrm{~h}$ & $0.36 \mathrm{~b}$ & $0.14 \mathrm{i}$ \\
\hline 19 & Edamame & $4.28 \mathrm{e}$ & $4.18 \mathrm{n}$ & $2.26 \mathrm{~s}$ & $0.65 \mathrm{r}$ & $0.55 \mathrm{~s}$ & $9.17 \mathrm{~h}$ & $13.3 \mathrm{c}$ & $4.23 \mathrm{~s}$ & $5.07 \mathrm{p}$ & $0.00 \mathrm{~s}$ & $0.47 \mathrm{e}$ & $0.31 \mathrm{q}$ & $0.53 \mathrm{e}$ & 0,13 & $0.00 \mathrm{n}$ \\
\hline
\end{tabular}

The fresh weight of the sprouts and the percentage of germination decreased with increasing drought stress. High osmotic pressure causes a decrease in water uptake by seeds which results in a low percentage of germination [22], [23]. Gepak kuning has a high germination value $(100 \%)$ to drought stress - $0.41 \mathrm{MPa}$ and

Root length, hypocotyl length, and the ratio of hypocotyl length and root length were significantly different between treatment without PEG and with PEG administration. This was also reported by Anwer et al. [24]. stated that the root length of Anthxanthun odoratum decreased with increasing PEG-6000 concentration in the germination phase.

According to Basal et al. [25] root parameters can be used as selection criteria for cotton cultivars for drought resistance in the germination phase, but cannot be used in peanut (Arachis hypogeal L.) [26]. Gepak Kuning has a root length that is significantly longer than other varieties, the higher the stress, the shorter the root length.

The level of tolerance of soybean varieties to drought stress is determined based on the calculation of the drought sensitivity index (DSI) on several drought variables. The drought sensitivity index value (DSI) is the relative value of the decrease in the value of various variables observed in stress conditions compared to nonstress conditions (control). The level of tolerance of each variety to drought stress was determined by looking at the consistency (highest number) of group classification of the drought sensitivity index value on the 6 germination variables (Table 3). The soybean variety that is included in the drought-tolerant group is the Dieng variety. Four of the six variables of germination had an index value of $\mathrm{S} \leq 0.5$. While the varieties included in the medium group with an DSI value on some of the germination variables between $0.5 \leq \mathrm{S} \geq 1$. Table 3 shows that the sensitivity index shows that the Gepak Kuning variety has a high level of tolerance to dryness stress compared to the other 18 varieties. Somewhat tolerant of drought include Detam 2, Dering 1, Deja 1, Detap 1, Detam 4, Devon 1, Dena 1, Derap 1, and Detam 3 while sensitive to drought include: anjasmoro, Dega 1, Grobogan, Detam 1, Dena 2 , Willis, Edamame, Devatra 1 and Devatra 2. The Detam 1 variety has the lowest value in several benchmarks, this is because the Detam 1 variety is a drought-sensitive variety [27]. 
Table 3. The Drought Tolerance Level of 19 Soybean Varieties Based on the Drought Sensitivity Index Value of Several Variables

\begin{tabular}{|c|c|c|c|c|c|c|c|}
\hline Varieties & $\begin{array}{c}\text { Hypocotyl } \\
\text { Length }(\mathbf{c m})\end{array}$ & $\begin{array}{c}\text { Root Length } \\
\mathbf{( c m})\end{array}$ & $\begin{array}{c}\text { Ratio } \\
\text { H/R }\end{array}$ & $\begin{array}{c}\text { Sprout } \\
\text { Weight (g) }\end{array}$ & $\begin{array}{c}\text { Germination } \\
\text { Persentase (\%) }\end{array}$ & $\begin{array}{c}\text { Indeks } \\
\text { Vigor }\end{array}$ & DSI \\
\hline Detam 2 & 0,85 & 1,02 & 0,76 & 0,71 & 0,31 & 0,57 & $\mathrm{M}$ \\
\hline Dering 1 & 0,97 & 1,16 & 0,91 & 1,01 & 0,31 & 0,11 & $\mathrm{M}$ \\
\hline Deja 1 & 1,01 & 1,00 & 0,98 & 0,98 & 0,00 & 0,23 & $\mathrm{M}$ \\
\hline Detap 1 & 1,02 & 0,76 & 1,02 & 1,04 & 0,31 & 0,91 & $\mathrm{M}$ \\
\hline Detam 4 & 0,89 & $-0,24$ & 0,94 & 0,87 & 0,92 & 1,37 & $\mathrm{M}$ \\
\hline Anjasmoro & 0,95 & 2,36 & 0,61 & 0,90 & 0,92 & 1,48 & $\mathrm{~S}$ \\
\hline Devon 1 & 0,96 & 0,65 & 0,96 & 0,83 & 0,00 & 0,23 & $\mathrm{M}$ \\
\hline Dena 1 & 1,04 & 0,05 & 1,08 & 0,78 & 0,00 & 0,80 & $\mathrm{M}$ \\
\hline Dega 1 & 1,03 & 1,06 & 1,01 & 1,04 & 0,61 & 1,82 & $\mathrm{~S}$ \\
\hline Grobongan & 1,02 & 0,93 & 1,01 & 1,00 & 0,92 & 1,71 & $\mathrm{~S}$ \\
\hline Derap 1 & 1,01 & $-0,03$ & 1,06 & 1,01 & 0,31 & 1,37 & $\mathrm{M}$ \\
\hline Detam 3 & 1,00 & 0,17 & 1,03 & 0,97 & 0,00 & 0,57 & $\mathrm{M}$ \\
\hline Detam 1 & 1,07 & 0,49 & 1,10 & 1,08 & 2,76 & 2,05 & $\mathrm{~S}$ \\
\hline GepakKuning & 0,92 & $-1,35$ & 1,03 & 1,16 & 0,31 & 0,11 & $\mathrm{~T}$ \\
\hline Dena 2 & 1,05 & 0,49 & 1,07 & 1,11 & 1,53 & 1,48 & $\mathrm{~S}$ \\
\hline Wilis & 1,01 & 2,28 & 1,11 & 0,85 & 1,23 & 1,82 & $\mathrm{~S}$ \\
\hline Devatra 2 & 1,04 & 2,56 & 0,80 & 1,20 & 2,45 & 0,91 & $\mathrm{~S}$ \\
\hline Devatra 1 & 1,10 & 2,15 & 1,02 & 1,34 & 0,00 & 0,46 & $\mathrm{~S}$ \\
\hline Edamame & 1,04 & 3,93 & 1,02 & 0,84 & 6,13 & 1,02 & $\mathrm{~S}$ \\
\hline
\end{tabular}

$\mathrm{T}=$ Tolerant, $\mathrm{M}=$ Medium, $\mathrm{S}=$ Sensitive

Gepak Kuning (tolerant)

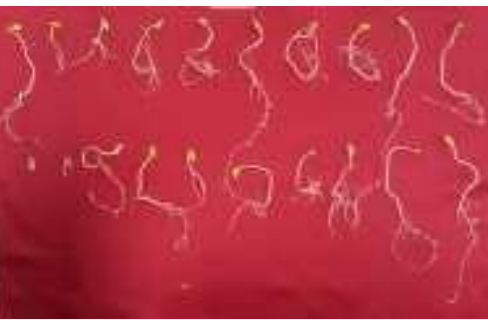

Dering 1(medium)

$5 \%$

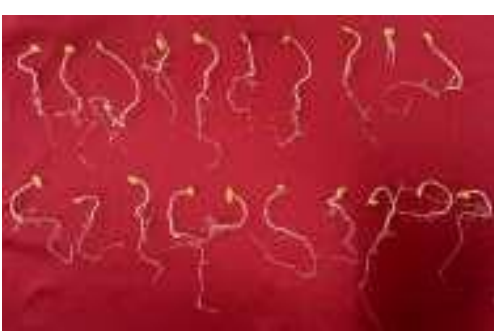

$10 \%$

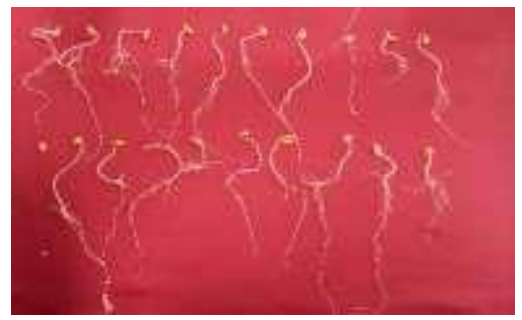

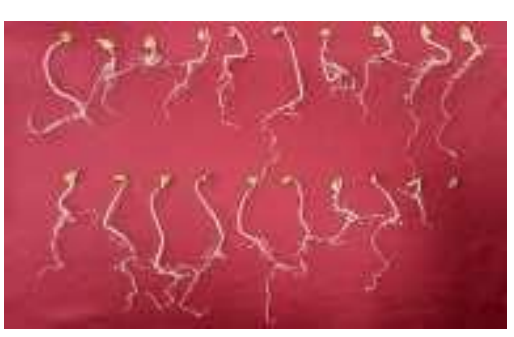

$15 \%$
Dena 2 (sensitive)
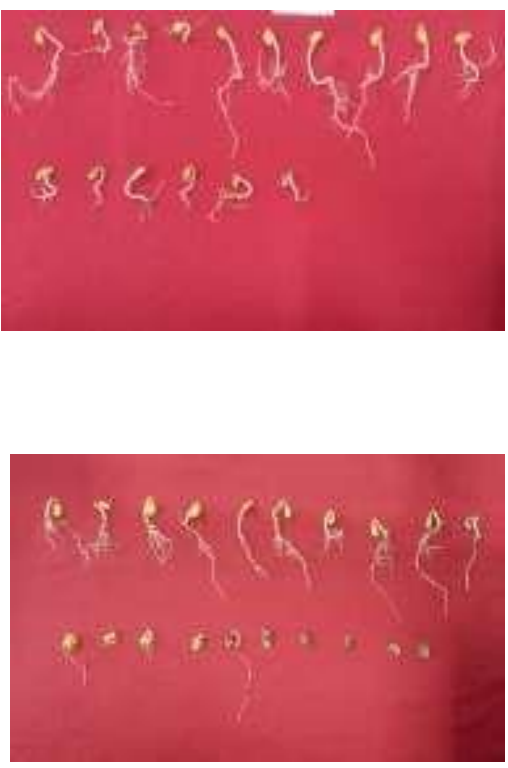

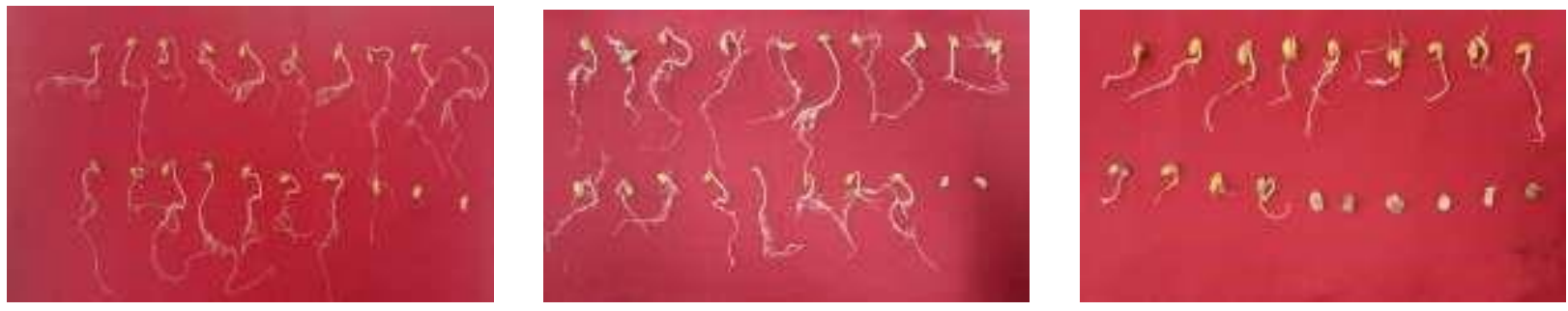

$20 \%$
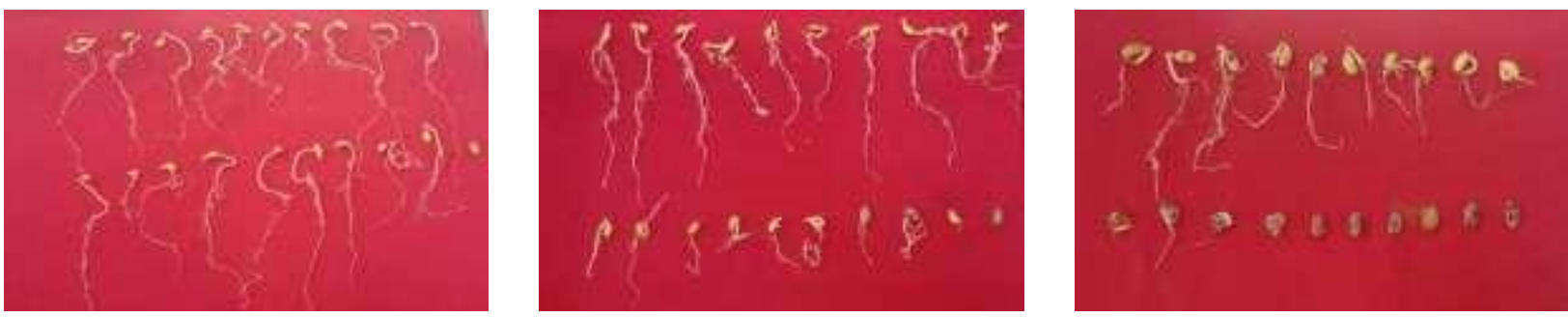

Figure 1. The performance of 3 soybean varieties with various PEG concentrations of 5\%, 10\%, $15 \%$ and $20 \%$.

One of the mechanisms for environmental resistance such as response to drought stress is to avoid or escape from these stress conditions. The morphophysiological mechanism of plants to avoid drought stress is the ability of plants to extend their roots to seek water sources far from the soil surface when drought stress occurs in areas near the soil surface [28]. Lestari's research [21] states that each seed has a different tolerance to drought due to differences in the morphological, physiological, biochemical, and molecular mechanisms of the seed. Hanum [29] argues that the effect of drought stress on soybean plants depends on the variety, size and duration of stress, and the period of plant growth, the level of tolerance of plants to drought stress can be determined by observing root development to distinguish between resistant plants and plants that are sensitive to drought.

\section{CONCLUSION}

1. The higher the PEG concentration, the lower the root length, hypocotyl length, ratio of hypocotyl length/root length, vigor index, germination percentage, and germination fresh weight.

2. The sensitivity index shows that the Gepak Kuning variety has a high level of tolerance to dryness stress compared to the other 18 varieties. Somewhat tolerant of drought include: Detam 2, Dering 1, Deja 1, Detap 1, Detam 4, Devon 1, Dena 1, Derap 1, and Detam 3 while sensitive to drought include: anjasmoro, Dega 1, Grobogan, Detam 1, Dena 2, Willis, Edamame, Devatra 1 and Devatra 2.

\section{ACKNOWLEDGMENT}

This work was supported by Excellent research scheme from Universitas Bengkulu with contract number: 2006/UN30.15/PG/2020.

\section{REFERENCES}

[1] Suprapto, 'Soybean and how to crops', Puslitbangtan, Bogor, 2000.

[2] Ministry of Agriculture of the Republic of Indonesia, 'Soybean production by province, 20142018'. 2019.

[3] T. Sudaryanto, and D. K. S. Swastika, 'Soybean economy in Indonesia', Agricultural Research and Development Agency. Food Crop Research and Development Center, Bogor, 2007.

[4] Research Institute for Various Nuts and Tubers, 'Description of superior soybean varieties 19182016', Research Center for Various Plants of Nuts and Tubers, Malang, 2016.

[5] Suhartina, 'Description of superior nuts and tubers varieties', Research Center for Various Plants of Nuts and Tubers, Malang, 2005.

[6] Y. J. Kim, S. Shanmugasundaram, S. J. Yun, H. K. Park, and M. S. Park, 'A simple method of seedling screening for drought tolerance in soybean', Korean J. Crop. Sci., vol. 46, pp. 284-288, 2001.

[7] L. Van den Berg, and Y. J. Zeng, 'Response of South African indigenous grass species to drought stress induced by polyethylene glycol (PEG) 6000', Afr. J. Bot., vol.72, pp. 284-286, 2006.

[8] L. Radhouane, 'Response of Tunisian autochthonous pearl millet (Pennisetum glaucum (L.) R. Br.) to drought stress induced by 
polyethylene glycol (PEG) 6000', African Journal of Biotechnology, pp.1102-1105, 2007.

[9] J. Levitt, 'Responses of plants to environmental stresses, vol. I chilling, freezing, and high temperature stress', Academic Press., London, New York, Toronto, 1980.

[10] M. Kulkarni, and U. Deshpande, 'In vitro screening of tomato genotypes for drought resistance using polyethylene glycol', African Journal of Biotechnology, vol. 6, pp.691-696, 2007.

[11] A. Guóth, I. Tari, A. Gallé, J. Csiszár, L. Cseuz, and L. Erdei, 'Changes in photosynthetic performance and $\mathrm{ABA}$ levels under osmotic stress in drought tolerant and sensitive wheat genotypes', Acta Biologica Szegediensis, vol. 52, no.1, pp. 91-92, 2008.

[12] J. E. Muller, and M. S. Whitsitt, 'Plant cellular responses to water deficit', Plant Growth Regulation, vol.20, pp. 119-124, 1996.

[13] J. J. Mullahey, S. H. West, and J. A. Cornell, 'Effects of simulated drought by polyethylene glycol bahia grass germination', Seed Science Technology, vol. 24, pp. 219-224, 1996.

[14] R. Hajiboland and B. Hasani, 'Responses of antioxidant defence capacity and photosynthesis of bean (Phaseolus vulgaris L.) plants to copper and manganese toxicity under different light intensities', Acta Biol. Szeged, vol. 51, pp. 93-106, 2007.

[15] M. Boydak, H. Dirik, F Tilki, and M. Calikoglu. Effects of water stres on germination in six provenances of Pinus brutia seeds from different bioclimatic zones in Turkey', Turkey Journal of Agriculture Forrest, vol. 27, pp. 91-97, 2003.

[16] H. Pirdashti, T. Z. Sarvestani, G. H. Nematzadeh, and A. Ismail, 'Effect of water stress on seed germination and seedling growth of rice (Oryza sativa L.) genotypes', Pakistan Journal of Agronomy, vol. 2, no.4, pp. 217-222, 2003.

[17] G. Okcu, M. D. Kaya, and M. Atak, 'Effects of salt and drought stresses on germination and seedling growth of pea (Pisum sativum L.)', Turkey Journal of Agriculture Forrest, vol. 29, pp. 237-242, 2005.

[18] G. L. Bonvissuto and C. A. Busso, 'Germination of grasses and shrubs under various water stress and temperature conditions', International Journal of Experimental Botany, vol. 76, pp. 119-131, 2007.
[19] U. S. Gupta, 'Crop improvement for Stress olerance', Science Publication Inc., USA, 1997.

[20] A. Blum, 'Crop responses to drought and the interpretation of adaptation', Plant Growth Reg., vol. 20, pp. 135-148, 1996.

[21] E. G. Lestari, and I. Mariska, 'Identification of drought-resistant Gajah mungkur, Towuti and IR64 rice somaclones using polyethylene glycol', Agronomy Bulletin, vol. 34, no.2, pp. 71-78, 2006.

[22] C. H. S. G. Menenses, R. L. A. Bruno, P. D. Fernandes, W. E. Pereira, L. H. G. M. Lima, M. M. A. Lima, and M. S. Vidal, 'Germination of cotton cultivar seeds under water stress induced by polyethyleneglycol-6000', Crop Science, vol. 68, no. 2, pp. 131-138, 2011.

[23] R. E. Brevadan, M. G. Klich, E. E. Sanchez, and M. N. Fioretti, 'Effects of water stress ongermination and seedling growth of ovegrass species', Plant Physiology and Growth, vol. 774, no. 7, pp. 35-36, 2012.

[24] M. Anwer, T. Mcnelly, and P. D. Purwain, 'Effect of polyethylene glycol on the growth of two populations of Anthxanthum odoratum', International Journal of Agriculture and Biology, 2004.[Online] Available: http://www.ijab.org.

[25] H. Basal, C. W. Smith, P. S. Thaxon, and J. K. Hemphill, 'Seedling drought tolerance in upland cotton', Crop Sci., vol. 45, pp. 766-771, 2005.

[26] R. Adisyahputra, Indrayani, and D. Eldina, 'Characterization of the properties and tolerance to dry stress of peanut (Arachis hypogea L.) national varieties at the germination stage', Journal of Mathematics, Science and Technology, vol.5, no.1, pp. 116, 2004.

[27] Balai Pengkajian Teknologi Pertanian Provinsi Sulawesi Selatan (BPTP), 'Research and assessment reports', Center of the study and Development of Agricultural Technology, 2010.

[28] F.C. Onwueme, 'The tropical tuber crops, yams, cassava, sweetpotato, and coco yams', John Wiley and Sons Chichester, New York, 1978.

[29] C. Hanum, Q. Muqnisjahw, S. Yahya, D. S. Sopandy, S. K. Idris, and A. A. Sahar, 'Growth of soybean roots on aluminum stress, drought and aluminum double stress and drought', Agritrop, vol. 26, no.1, pp. 13-18, 2007. 